\title{
Prácticas de lectura y escritura con potencial transformativo. Una investigación colaborativa en contextos escolares, hospitalarios, comunitarios y barriales
}

\author{
Reading and Writing Practices with a Potential for Transformation. \\ Collaborative Research in School, Hospital, Community and Working- \\ Class Neighborhood Environments
}

DOI: https://doi.org/10.32870/dse.v0i23.953

\author{
Ana Inés Heras* \\ María Amalia Miano** \\ Ana Rosa Moyano*** \\ Mariel Lucía Ferreira**** \\ Eliana Verónica Soto***** \\ Horacio Cárdenas****** \\ Silvina Adriana Esposito***** \\ Judith del Valle Martínez ${ }^{* * * * *}$ \\ María Inés Mori******* \\ María Laura Galli******** \\ María Victoria Morana******** \\ Ana Patricia Schneider**********
}

\begin{abstract}
Resumen
En este escrito desarrollamos el marco conceptual de un proyecto de investigación colaborativa denominado Prácticas de Lectura y Escritura con Potencial (Trans)Formativo, para lo cual explicamos primero la perspectiva que se asumió al inicio del proyecto y sus transformaciones a lo largo de tres años de trabajo exploratorio. A continuación, analizamos cuatro prácticas (trans)formativas, ubicadas en ámbitos diversos de la Ciudad de Buenos Aires. Hemos identificado estas prácticas porque, tomadas en su conjunto, nos permiten: a) mostrar la noción de potencial transformativo cuando se lo piensa ligado a prácticas de lectura y escritura; b) describir y analizar la centralidad que ocupan el leer y el escribir en espacios, organizaciones e institucionalidades muy diferentes entre sí, pero que tienen en común un potencial para interpelar el orden dado y transformarlo, y c) hacer visible cómo estas prácticas fueron apelando a diversos formatos, soportes, disciplinas y lenguajes, y al hacerlo, reconfiguraron el mismo sentido de leer y escribir. En la sección final subrayamos los alcances de nuestro estudio en tanto los análisis de experiencias singulares
\end{abstract}

\footnotetext{
* Doctora en Educación. Líneas de investigación: etnografía, sociolingüística y procesos de aprendizaje. Investigadora, Instituto para la Inclusión Social y el Desarrollo Humano y de la UNSAM. Argentina. aheras@unsam.edu.ar

** Doctora en Ciencias Sociales. Líneas de investigación: educación rural, cogestión, etnografía, prácticas de lectura y escritura, sociolingüística de la interacción. Investigadora, Instituto para la Inclusión Social y el Desarrollo Humano y Laboratorio de Investigación en Ciencias Humanas (LICH-UNSAM-CONICET). Argentina. amiano@unsam.edu.ar

*** Licenciada en Letras. Líneas de investigación: lectura y escritura en distintos contextos, didáctica de la lectura y la escritura literaria. Profesora-investigadora, UNSAM. Argentina. anarmoyano@yahoo.com.ar

**** Estudiante de la licenciatura en Estudios de la Comunicación. Líneas de investigación: prácticas de lectura y escritura. UNSAM. Argentina. mferreira@estudiantes-unsam.edu.ar

***** Licenciada y profesora en Letras. Líneas de investigación: prácticas de lectura y escritura. UNSAM. Argentina. elianaverosoto@hotmail.com

***** Profesor de Enseñanza Primaria en escuelas del sur de CABA, Instituto de Educación Superior Juan B. Justo, Escuela Normal Superior. Líneas de investigación: didáctica de la lengua y matemáticas en la escuela primaria. Argentina. cardenashoracio@yahoo.com.ar
} 
Ana Inés Heras, María Amalia Miano, Ana Rosa Moyano, Mariel Lucía Ferreira, Eliana Verónica Soto, Horacio Cárdenas, Silvina Adriana Esposito, Judith del Valle Martínez, María Inés Mori, María Laura Galli, María Victoria Morana y Ana Patricia Schneider

nos permiten arribar a conclusiones en relación con el potencial transformativo de la lectura y la escritura. Asimismo, dejamos planteadas algunas limitaciones del estudio realizado e interrogantes para desarrollo futuro.

Palabras clave: prácticas de lectura y escritura - Buenos Aires - hospitales - escuelas -organizaciones comunitarias.

\begin{abstract}
In this piece we develop a conceptual framework for a collaborative research project on Literacy Practices and their Potential for Transformation. We first explain our theoretical and methodological approach, describing how we constructed a relevant framework over three years of exploratory work. We then analyze four different experiences currently being conducted in the City of Buenos Aires, Argentina. We have identified this set of practices because, taken together, they allow us to a) show the notion of a potential for transformation when linked to reading and writing practices, b) to describe and analyze the central role of reading and writing in very different spaces, organizations and institutions with a common potential to address the order established and transform it, and c) to make visible how these practices appealed to different formats, supports, disciplines, and languages, and in doing so reshaped the very meaning of reading and writing. We conclude by underscoring the scope of our study insofar as the analysis of specific experiences leads us to insights on the transformational potential of reading and writing practices, and point out some of the limitations of our study and possible lines of further research.
\end{abstract}

Key words: eading and writing practices - Buenos Aires - hospitals - schools - social and community organizations.

En primer lugar, agradecemos a todos y todas las personas involucradas en las experiencias con las que hemos trabajado. En segundo lugar, comunicamos que el trabajo reportado aquí ha sido sostenido por el financiamiento del Programa "Humanidades Investiga" de la Universidad Nacional de San Martín, Escuela de Humanidades, y por el Programa “Aprendizaje de y en Autogestión", de inserción doble en la UNSAM y el Instituto para la Inclusión Social y el Desarrollo Humano. Asimismo, el trabajo realizado para este artículo ha permitido que se generen antecedentes de investigación para crear la Maestría en Literatura Infantil y Juvenil, en trámite ante CONEAU con antecedente en la Especialización en Literatura Infantil y Juvenil.

\title{
Introducción
}

\section{El potencial de transformación y su relación con la lectura y la escritura}

Las prácticas vinculadas a la lectura y escritura que analizamos aquí, realizadas por diferentes grupos, funcionan en instituciones sociocomunitarias o lugares que pertenecen al Estado en la

\footnotetext{
***** Posgrado en Residencia de Salud Mental y Residencia de Educación para la Salud. Adscrita a Pediatría del Hospital General de Agudos “Dr. Enrique Tornú". Interdisciplina en el hospital público. Argentina. silesposito@hotnail.com

****** Licenciada en Musicoterapia. Adscrita a Pediatría del Hospital General de Agudos "Dr. Enrique Tornú". Interdisciplina en el hospital público. Argentina.judithdelvallemartinez@gmail.com

******* Bibliotecaria especialista docente de Nivel Superior en Educación y TIC. Coordinadora de la Biblioteca de la Escuela Normal Superior N 7 "José María Torres". Argentina.mainemori@gmail.com

******* Licenciada en Psicopedagogía. Líneas de investigación: lectura y escritura en la primera infancia. Adscrita al Programa Primera Infancia-GOIE-SSCPEE-Ministerio de Educación e Innovación-GCBA. Argentina. marialauragalli@yahoo.com.ar

********* Profesora de Educación Inicial. Forma parte del Programa Niñez y Adolescencia, Ministerio de Cultura de GCBA. Interdisciplina en el hospital público. Argentina. mvmorana@gmail.com

********* Diplomada en Psicomotricidad en Educación. Escuela Domiciliaria № 1 (Área Educación Especial, CABA). Interdisciplina en el hospital público. Argentina. patosoloescuela@gmail.com
}

Diólo gos

sobre Educación

año 12 | número 23 | julio-diciembre 2021 | ISSN 2007-2171 
Ciudad de Buenos Aires (como escuelas, hospitales públicos, entre otras); se sitúan en contextos donde los derechos de quienes participan de las experiencias están continuamente vulnerados en lo que respecta a su salud, educación, vivienda, trabajo, alimentación y recreación. Al exponerlas, damos cuenta de cómo estas van produciendo modos diversos de interpelar esas condiciones de existencia en que se encuentran los sujetos (estudiantes, usuarios del sistema de salud pública y participantes de experiencias sociocomunitarias). Por ello, permiten plantear otras lógicas sociales posibles. Así, presentamos un análisis de prácticas de lectura y escritura cuyo propósito explícito es promover la transformación social.

En tanto son prácticas específicamente propuestas en torno a la lectura y la escritura, podría decirse que su espacio de acción es restringido en relación con la transformación social. Sin embargo, propondremos que construyen un modo de leer el mundo en el sentido otorgado por Freire (1983) y, por lo tanto, permiten crear formas diversas de vivir e imaginar. Nuestra pregunta general de análisis interpretativo ha sido: ¿Cuáles son los modos contextualmente situados en que cada una de estas experiencias sostiene y desarrolla un potencial de transformación, tanto en el grupo que la lleva adelante como en relación con el contexto organizacional en que se alojan?

Nuestra perspectiva pone a debate la premisa de que existe una lectura correcta; la posición que tomamos es que se construyen diferentes modos de producir sentido, así como también diversas maneras de constituirse los grupos singulares en comunidades de lectura 0 de escritura. Estas prácticas, desarrolladas y conceptualizadas de este modo, se encuentran en tensión con otras que asumen diferentes nombres y sentidos según el contexto, entre ellas, la noción de que la comprensión lectora supone una lectura e interpretación única de cualquier texto que se aborde, o la noción de estricta decodificación de la letra como sinónimo exclusivo de lectura. De esta manera, es posible identificar situaciones donde algunas dicotomías asentadas en esas interpretaciones restringidas, que generan clasificaciones polares (p.e., letrado/ iletrado), no alcanzan a describir las realidades que tienen lugar en relación con el uso de la lectoescritura. Se amplía así el campo de observación y análisis a diversas herramientas concurrentes para la producción de sentido y de construcciones simbólicas, diversificadas y mediadas por una variedad de soportes y formatos (p.e., imágenes fijas y móviles, textos audiovisuales, objetos, escritura en sentido tradicional del término, otros tipos de escrituras).

Concebir de este modo la lectura y la escritura implica que, para comprender cualquiera de las prácticas desarrolladas en los proyectos que se analizan aquí, precisamos distinguir la complejidad en que se desarrollan. Proponemos que pueden comenzar a analizarse teniendo en cuenta las materialidades y las interacciones involucradas en ellas.

Por otra parte, en los contextos que hemos documentado, la lectura y la escritura se desarrollan en conjunto con potencias de creación artística y recreativa ligadas al ejercicio de la imaginación como capacidad humana distintiva. En nuestro trabajo, esta vinculación entre leer, 
escribir y crear se produce además en organizaciones variadas (escuelas, hospitales, organizaciones barriales, bibliotecas, entre otras) y en relaciones interpersonales que ponen en una nueva relación los roles inicialmente asumidos (docentes, alumnos, médicos, psicólogas, trabajadoras sociales, investigadoras de ciencia y técnica, etc.). Así, se producen transformaciones en las prácticas mismas, en los sujetos que las realizamos y en las organizaciones que nos alojan.

El desarrollo del artículo plantea a continuación una sección de encuadre conceptual y metodológico. En él ubicamos al lector/a en el marco de trabajo de los Nuevos Estudios de Literacidad y de investigación colaborativa, y damos cuenta de los criterios de análisis e interpretación que utilizamos. Hemos tomado las nociones de potencial formativo, potencial transformativo y potencial performativo vinculándolas al concepto de literacidad (Zavala, Niño-Murcia, Ames, 2004; Zavala, 2002). Enseguida, en el apartado de Desarrollo se presenta una descripción analítica de las prácticas seleccionadas; a medida que se expone cada experiencia se puntualizan los hallazgos que permitirán dar cuenta de las respuestas a la pregunta analítica general. Luego, en la sección de Resultados presentamos una discusión de las dimensiones abordadas para dejar planteadas las cuestiones relevantes que surgen de nuestro análisis. Explicaremos también potencialidades, limitaciones e interrogantes que permanecen aún sin responder, a modo de indicar posibilidades para la construcción de agendas de investigación que involucren a los sectores comunitarios, docentes, académicos y de la función pública.

En este escrito usaremos el pronombre nosotros para referirnos a un plural de autoría que responde a una escritura de 12 personas que, al trabajar en conjunto, fuimos generando un marco conceptual compartido y proponiendo modos de analizar el trabajo realizado por cada uno/a en la producción de una escritura colectiva, para dar lugar a este artículo. Cabe aclarar también que el trabajo de escritura se lleva adelante en el marco de un proyecto de investigación colaborativa de propiedad colectiva (Heras, 2014). La iniciativa comenzó en una universidad del conurbano bonaerense, a partir del trabajo de dos equipos de docentes e investigadores/as radicados allí. Varios/as de nosotros además habíamos venido trabajando con grupos autoorganizados en el marco de iniciativas de política pública estatal para la promoción de la lectura y la escritura. Además, algunos de esos profesores/as somos miembros o participantes directos de varias de las experiencias, sea como vecinos/as, como coordinadores/as de actividades y/o como profesionales. La idea del proyecto en el cual la escritura de este trabajo tiene lugar se diseñó en 2014 y se escribió y puso a consideración de la universidad en 2015.

A partir de 2016, se fueron desarrollando algunas actividades preliminares (contactos, armados de redes de relaciones entre experiencias, un primer relevamiento exploratorio) y las actividades de vinculación con las experiencias comenzaron en 2017, ya que el Programa Humanidades Investiga se aprobó durante ese año. El proyecto continúa, aunque formalmente el apoyo recibido de la universidad ya terminó. Sin embargo, en tanto parte de lo realizado, permitió consolidar el Programa de Maestría en la UNSAM, y que el Instituto para la Inclusión 
Social y el Desarrollo Humano, a través del Programa Aprendizaje de y en Autogestión, continúe realizando aportes para sostener la tarea; nuestro trabajo continuará en el tiempo. Durante estos años de desarrollo del proyecto se llevaron a cabo diferentes actividades de documentación -complementarias entre sí- en las que participan quienes, desde las experiencias y la universidad deseamos hacerlo, siguiendo para ello algunos lineamientos acordados que se explicarán más adelante.

\section{Leer y escribir como prácticas sociales. Surgimiento y aportes}

de los Nuevos Estudios de la Literacidad

En el campo de los estudios de la literacidad, durante los años de 1980 se desarrolló una línea de pensamiento que cuestionó la visión, preponderante en ese momento, que distinguía dicotómicamente entre oralidad y escritura, perspectiva que ya hace más de dos décadas ha venido sosteniendo el enfoque de los Nuevos Estudios de la Literacidad (NEL en adelante) (véase Barton, Hamilton, 1998; Gee, 1986; Kalman, 2003; Scollon, Scollon, 1981; Zavala, 2002). De este modo, la perspectiva de los NEL ha permitido identificar procesos de escritura y lectura como situaciones de construcción de sentido que se sustentan en la interacción, tanto entre seres humanos como entre seres humanos y objetos, artefactos, representaciones, según dimos cuenta en trabajos anteriores (Heras, Miano, 2018; Miano, Heras, 2015, 2018). Inclusive, en los últimos años este marco conceptual ha permitido comprender y desarrollar modos de intervenir en la enseñanza escolar de distintas áreas curriculares a partir de interrogar qué es leer y escribir como práctica situada contextualmente (López Bonilla, 2013; López Bonilla, Tinajero, Pérez Fragoso, 2006).

Si bien esta línea de trabajo había comenzado a perfilarse, por un lado en el área de la lingüística aplicada y por otro en la antropología y sociología, como campos distintos entre sí, tomaba como objeto de estudio a la lengua o a las lenguas en sí mismas. Fue entre finales de los años 1970 y comienzos de 1980 que esos campos comenzaron a referenciarse entre ellos y se empezaron a realizar trabajos de investigación en sitios distintos a las escuelas: organizaciones sociocomunitarias, barrios, interacciones en las calles, entre otros, precisamente porque la conceptualización sobre qué es leer y escribir amplió su panorama y construcción de objetos de estudio. Lo que comenzaba a constituirse en ese momento era la posibilidad de pensar la escritura y la lectura como situaciones que se definen contextualmente, donde las prácticas concretas podrían informar y sostener la capacidad de interactuar con la materialidad específica (la letra escrita, la letra hablada, los números o formas de pensar, las cantidades, etc.) para producir sentido. Así, en el campo de la lectura y la escritura, entre 1980 y el presente, se desarrolló una variedad de enfoques, por un lado, y de investigaciones empíricas, por otro, que permitieron poner a discusión qué se entiende por leer y escribir, y qué cuenta entonces como "leer / escribir" en cada contexto singular (por ejemplo, véase Rockwell, 1992, 2006, 2012; Heras, Green, 2010; SBCDG, 1992, 1995). 
También en matemáticas ocurrió algo similar, e incluso se desarrollaron nuevos conceptos y campos de estudio tales como el concepto de numeracy o math literacy, en inglés, o el entonces nuevo campo de street maths como un área de estudios (por ejemplo, Barta, Brenner, 2009; Frankenstein, 1989; Mills, 1991; Weissglass, 2001). Inclusive Marilyn Frankenstein propuso hablar de alfabetización matemática crítica, una perspectiva similar a la de alfabetización crítica y alfabetización popular en América Latina; y Hirsch-Dubin (2009) desarrolló una serie de conceptos específicos para comprender los modos matemáticos embebidos en prácticas ancestrales mayas, vigentes hoy en México y Guatemala, que no habían sido reconocidos como conocimientos matemáticos dado el marco conceptual usado hasta el momento, y que el concepto de etnomatemáticas permitió hacer visibles.

En los momentos iniciales de esos desarrollos se produjeron préstamos y fertilizaciones conceptuales entre disciplinas e investigadores de perspectivas diferentes. Por ejemplo, Scribner y Cole (1981) ligaron el estudio de la lectura y la escritura al campo de los estudios cognitivos, porque comenzaron a identificar que no se trataba de mayor o menor inteligencia, o de coeficiente intelectual, sino de cómo ciertas prácticas culturales conformaban modos de pensar, hacer, creer y observar. Más tarde, Rockwell (2006) vinculó el uso de la lectura a las formas de percibir la relación con la tierra de algunas comunidades campesinas en América Latina y dio a conocer la compleja efectuación de traducciones (simbólicas, lingüísticas y de soportes, por ejemplo, entre un texto y un mapa o entre un mapa y un relato oral) que son necesarias en ciertos contextos campesinos. Todo esto puso en cuestión qué cuenta allí como "ser letrado/a".

De este modo, y junto a otros estudios que se realizaron en ese momento y en décadas posteriores en el campo de las etnografías educativas, se comenzó a distinguir que ciertas habilidades (como abstraer, razonar silogísticamente o descontextualizar algo) no eran producto de capacidades cognitivas o de la alfabetización, sino de modos apropiados de actuar y comprender en contexto. Así, las aulas escolares comenzaron a ser estudiadas desde esta perspectiva de la generación de conocimiento contextualmente situado (Craviotto, Heras, 2001; Floriani, 1993; Fránquiz, Green, Craviotto, 1993; Heras, 1993, 1995; Heras, Craviotto, Espíndola, 1999) y también los estudios producidos en el Departamento de Investigaciones Educativas en México, entre otros.

Otros estudios etnográficos, ligados ya más directamente a la lectura, la escritura y la perspectiva crítica y popular, comenzaron a poner de relieve que existen distintos modos de producir conocimiento a través de leer, escribir e inclusive representar. Entre estos últimos estudios se identificó que distintas expresiones (el teatro, la música popular y las artes plásticas) configuran modos diversos de producir lecturas del mundo (Broyles González, 1994; Heras, 1999), ampliando así lo que se configuró en el campo con el concepto de literacidad. El concepto de alfabetización (ligado estrictamente a la letra escrita) era definitivamente restringido para abarcar esta variedad de concepciones. 
Acompañando a estos estudios, se realizaron otros en campos afines que continuaron presentando evidencia sobre la importancia de definir la lectura y la escritura como prácticas situadas contextualmente; por ejemplo, etnografías educativas, etnografías de sectores específicos en ámbitos campesinos, y estudios de las multiplicidades de formatos y soportes asociados en lo que se entendía hasta el momento como alfabetización. Conviene entonces poner en foco, que fue durante un tiempo relativamente largo (cuarenta años) que el campo de los estudios de la literacidad se fue desarrollando con los aportes mencionados y en diálogo con otras disciplinas, tales como la estética, el arte, los estudios visuales y audiovisuales, los estudios culturales, entre los más influyentes (p.e. Cambre, 2013, Céspedes, Guarini, 1995; Fernández Polanco, 2016; Weschler, 2016). Algunos de estos estudios, además, abrieron discusiones al poner en tensión el concepto de literacidad y literacidad visual por considerarlo demasiado ligado a la lógica de la letra escrita, que se diferencia de la lógica de producción y comprensión visual, audiovisual y performativa.

Además, y como sabemos, los inicios del siglo XXI trajeron una inquietud fuerte hacia posiciones que integraran conocimientos de disciplinas diversas, con la consecuente interpelación a las metodologías únicas de los campos específicos. Fue en esos años cuando comenzó a posicionarse lo que tomó el nombre de Nuevos Estudios de Literacidad como tal, aun cuando hoy podamos pensar que el apelativo nuevo puede haber quedado antiguo. Lo que fue novedad en su momento, fue integrar disciplinas diversas y campos hasta entonces relativamente separados (etnografía, estudios literarios, estudios culturales, letras), de modo tal que pudieran realizar elaboraciones teóricas diferentes y construir objetos de indagación antes no identificados.

Los principales hallazgos de este campo fueron los ligados a la concepción de que las relaciones entre escritura y oralidad son complejas, que varían contextualmente, que inclusive una misma persona puede adoptar distintas formas en relación con la escritura y la lectura de acuerdo con contexto e interlocutores, y que, atravesando todas estas cuestiones, subyacen preguntas acerca de las relaciones de poder. Este aspecto es crucial para el campo, y ha sido profundizado por muchos autores/as de diferentes países y contextos (de habla francesa, inglesa, castellana, portuguesa, inicialmente, y luego, también de y en otras lenguas como las nativas de América: el wichi, guaraní, mapuche y aymara). Lo que interesa destacar como otro punto común a esta proliferación temática, lingüística y geográfica es que permitieron ratificar la tesis de que existe una pluralidad de formas letradas y, por lo tanto, cada una de dichas formas actúa y se valida en relaciones sociales, políticas, económicas y culturales contextualmente situadas (Bonnin, 2018). Surge como comprobación, además, que todas las formas de literacidad están dispuestas en un campo de posibles complementaciones y sinergias, pero también de disputas y tensiones acerca de la pertinencia, sentido, corrección o posibilidad de ser reconocidas como tales. Asimismo, dicho campo complejo evidencia que paulatinamente se han realizado cone- 
xiones, hibridaciones y acciones de complementación de géneros literarios y formas que en otros momentos de la historia habían estado más fuertemente separadas e institucionalizadas.

Es a partir de este marco conceptual que hemos documentado y analizado en conjunto una serie de prácticas en hospitales, escuelas, organizaciones comunitarias y sociales en la Ciudad de Buenos Aires. Un aspecto que resultó crucial fue, precisamente, tomar este marco conceptual de inicio para darnos la posibilidad de identificar prácticas que se desarrollan en contextos muy específicos que, sin embargo, cuentan como prácticas vinculadas al leer y escribir transformativas de modo abarcador, como argumentaremos más adelante.

Sin embargo, y como propondremos, cada uno de los ámbitos donde estamos documentando prácticas de lectura y escritura pueden constituirse como campos de desarrollo conceptual e investigativo por sí mismos; por ejemplo, prácticas de lectura y escritura en contextos hospitalarios, escolares, comunitarios o barriales. Nuestra documentación pone en evidencia que las prácticas de lectura y escritura pueden hacer visibles aspectos vinculados a las formas del poder que muchas veces transcurren en esos sitios, tanto las que se objetivan en maltratos hacia grupos de personas a quienes se considera como "faltas de" (faltas de cultura, alfabetización, desarrollo cognitivo, etc.), como aquellas formas que precisamente cuestionan esas jerarquías y se convierten, en su misma efectuación, en transformativas. El derecho a la palabra, en tensión con un tipo de poder hegemónico y dominante, parece construirse a través de un cierto tipo de prácticas de lectura y escritura que, como decíamos en el apartado anterior, permiten leer el mundo (en el sentido del contexto donde estamos situadas/os) e interpelarlo.

\section{Metodología}

En el campo de los estudios sobre la lectura y la escritura existen antecedentes sobre investigaciones colaborativas y reflexión sobre la propia práctica (véase, por ejemplo, la compilación de Frisch y Stoppani, 2014, y más recientemente, la compilación de Marucco, en prensa). Tomamos estas áreas para ubicar nuestra forma de trabajo en relación con ambos tipos de investigaciones puesto que el análisis presentado aquí ha sido realizado tanto en colaboración como a partir de la reflexión sobre la práctica de cada uno/a de los participantes. Además, aspiramos a realizar un aporte metodológico al campo de los estudios de la literacidad desde el ángulo de lo que se puede investigar en colaboración y reflexión de la práctica propia, simultáneamente.

En el año 2014, un equipo alojado en una universidad pública del conurbano bonaerense comenzó a diseñar una propuesta para documentar y analizar prácticas de lectura y escritura con potencial formativo. A finales de 2015 se presentó a la universidad, donde varios/as del equipo enseñamos y hacemos investigación. En esa formulación distinguimos que varios de quienes formábamos parte del equipo de investigación éramos a la vez trabajadores universitarios (investigadores) y participantes o coordinadores de experiencias de lectura y escritura en distintos contextos (escuelas, universidades, centros culturales, colectivos autoconvocados ba- 
rriales, prisiones, etc.). De esta manera, nuestro sujeto colectivo de investigación tiene la peculiaridad de ser una integración compleja de investigadores-activistas, estudiantes universitarios y docentes que queremos investigar sobre nuestras propias prácticas y, en conjunto con otros, también sobre sus prácticas.

Nuestro diseño inicial planteó identificar colectivos de trabajo cuya perspectiva sobre leer y escribir pudiera ser descrita como formativa, que en ese momento se definió en sentido amplio como prácticas de lectura y escritura, y que a la vez fueran específicas del campo literario, interpelaran el contexto en que se alojaban provocando transformaciones, pusieran a debate nociones del sentido común sobre "letrado / iletrado" o "lectura y escritura" y permitieran ser pensadas como acciones que generaban sentidos y discursos sobre la justicia y el acceso a derechos de poblaciones habitualmente sometidas o vulneradas.

Nuestro proyecto comenzó a implementarse entre 2016 y 2017, y hasta 2018 nos vinculamos con 25 experiencias de trabajo con lectura y escritura realizadas en CABA y el conurbano bonaerense, en las cuales sus participantes identificaban la orientación mencionada arriba. A partir del primer contacto en relación con este proyecto, establecimos relaciones diferentes con cada colectivo, con la salvedad de que el primer paso fue igual para todas las experiencias: realizamos una encuesta autoadministrada para, por un lado, relevar algunas posiciones con respecto a las nociones básicas que nuestro proyecto quería explorar, y por otro, indagar el grado de interés de los participantes de las experiencias para realizar un trabajo colaborativo.

A partir del establecimiento de estas relaciones, se generaron herramientas de documentación diversas que, en su conjunto, podemos describir del siguiente modo: entrevistas en profundidad con integrantes de las experiencias y organizaciones; audiograbación y transcripción completa de las mismas; revisión conjunta con los/las participantes de dichas entrevistas y diálogos posteriores al respecto; bitácoras de registro autoetnográfico por parte de los/las participantes, y etnográfico cuando se trabajaba con integrantes del equipo; registros visuales (fotografías, croquis, diagramas, dibujos) y audiovisuales. A partir de este acervo documental se fue trabajando con el material en reuniones sucesivas, en configuraciones variadas (es decir, por pequeños grupos y/o en el grupo ampliado), para distinguir núcleos de sentido reiterados entre las experiencias, diferencias singulares de cada experiencia, constructos explicativos generados por las propias experiencias en su autoetnografía y etnografía acompañada por los/las demás y sistematización de estos núcleos de sentido para generar categorías conceptuales a explorar. Más adelante se explicita este proceso.

Asimismo, y a través de una página web aún en construcción, se está mapeando geográficamente la ubicación de las experiencias y se las está referenciando entre ellas y poniendo en visibilidad (para sí mismas y para una audiencia ampliada). Las herramientas utilizadas fueron revisadas una y otra vez. Así, las encuestas y entrevistas que fueron construidas como herramientas de relevamiento en una primera instancia, sirvieron como base para el diálogo, la do- 
cumentación continua y el intercambio analítico que dio vida a este artículo y a otras escrituras colectivas en curso.

A poco tiempo de haber realizado el contacto con las experiencias y en diálogo con una de ellas en particular, surgió la inquietud acerca del concepto de potencial formativo y se sugirió cambiarlo por el de transformativo. Se explicitó que formativo, como adjetivo, parecía tener un sesgo moldeador, de arriba para abajo, como si una externalidad pudiese dar forma, educar, e inclusive, tal vez hasta ejercer cierta violencia de definición sobre prácticas que parecían tener un potencial más dinámico, incierto y abierto, y por ello, (trans)formador. Por otro lado, el concepto transformativo incluía la transformación en aquellos mismos que llevan adelante las prácticas como sujetos participantes por igual, y no en la clásica división de usuarios/profesionales o alumnos/docentes. Se tomó esta perspectiva para continuar el trabajo de relevamiento y análisis de las experiencias, sumándole también a esta noción la importancia del cuerpo en situación, ya que surgió del primer relevamiento inicial como un punto importante a atender. En la conceptualización de lo transformativo se tornó importante reconocer la potencia de la acción y del cuerpo en situación. Se tomó para ello el concepto de performativo (Butler, 2017).

Entre 2018-2020 se realizaron una serie de pasos recursivos:

- Identificación de prácticas por su carácter (trans)formativo / (per)formativo, tomando en cuenta los aportes realizados en conversaciones analíticas con los grupos.

- Generación de acuerdos de trabajo en relación con la noción de investigación colaborativa de propiedad colectiva en acto.

- Indagación de los sentidos que algunos de los participantes de estas prácticas dan a leer y a escribir (a través de una encuesta, entrevistas, observaciones, comunicaciones por mail y con instrumentos virtuales y reuniones pre-Covid19).

- Realización de transcripciones y audiomapas (mapeo del time-code por núcleo semántico, una técnica ya probada en Heras y Miano, 2014).

- Revisión y análisis de transcripciones y rescrituras de pensamientos, nociones y análisis de situaciones en equipo para la construcción de una metodología de autoría múltiple.

- Identificación de algunas categorías de análisis propuestas en diálogo, e identificación de frases que resultaron claves para guiar la comprensión de los sentidos que los participantes damos a las prácticas.

- Realización de reuniones periódicas de elaboración para poner en foco las cuestiones que se venían identificando y poder continuar produciendo algunas conceptualizaciones (además se realizaron reuniones de trabajo en torno a la construcción colectiva de una página web del proyecto). 
Los últimos tres pasos descritos ya habían sido probados en proyectos anteriores (por ejemplo, Heras et al., 2014).

\section{Desarrollo}

Análisis descriptivo: materialidades, sujetos, formatos, lenguajes e infraestructura

A partir de las acciones descritas en el apartado anterior, en un encuentro abierto con 25 participantes de las experiencias, se fueron distinguiendo núcleos de sentido. Denominamos núcleos de sentido a aspectos que los/las participantes de las experiencias identifican como formando parte de las prácticas de lectura y escritura que analizamos aquí y que mantienen entre sí relaciones complejas, ya que cada uno toma significado en relación con todos los otros núcleos. Identificamos los siguientes: sujetos que llevan adelante las prácticas; materialidades en que se construyen esas prácticas y que a la vez se crean en dicha interacción; formatos en que las prácticas de lectura y escritura se objetivan, y diversos lenguajes (orales, escritos, visuales, corporales) involucrados en las mismas; infraestructura donde se desarrollan las prácticas, que a su vez se modifican al irlas alojando. Junto a identificar y realizar un análisis descriptivo de estos núcleos de sentido, producimos una interpretación de las formas en que las prácticas de escritura y lectura parecían ir construyendo un potencial de transformación al llevarse adelante desde la perspectiva que mencionamos inicialmente, es decir, como interpelación y creación.

A continuación, se analizan cuatro experiencias que permitieron producir un análisis conjunto de su quehacer en encuentros, presenciales y virtuales, entre quienes escribimos este artículo y se decidieron a formar parte del colectivo de escritura para este texto. Es decir, de las 25 experiencias originalmente relevadas, hubo participantes de cuatro de ellas que quisieron involucrarse en la escritura de este trabajo, mientras que otros colectivos de escritura se siguen conformando para analizar distintos aspectos del material relevado. Las experiencias presentadas aquí se desarrollan en el ámbito público y se alojan en un hospital, una escuela primaria, una escuela secundaria y una escuela que tiene todos los niveles educativos (desde jardín de infantes a instituto terciario de enseñanza). De este modo, la diversidad de contextos es un hecho relevante para el análisis presentado aquí. A continuación, se describe analíticamente cada una de las experiencias en relación con los núcleos de sentido.

\section{Prácticas de lecturas y escrituras en el hospital}

Esta experiencia funciona en la sala de espera de un hospital público, en el sector de pediatría ambulatoria al que asisten familias que traen a sus niños y niñas para su seguimiento periódico de salud. Estas familias, en su mayoría, residen en barrios y asentamientos cercanos al hospital, mientras que otras provienen del conurbano bonaerense, principalmente de localidades de la zona oeste. En ese espacio, a lo largo del tiempo se constituyó la biblioteca "El rincón de los 
sueños", donde se realizan actividades de lectura y escritura, juegos con objetos, canciones y palabras, y actividades de expresión con el cuerpo.

Inicialmente, se trató de pequeños momentos que surgieron de modo espontáneo en la sala de espera como un modo diferente de esperar, que a lo largo del tiempo permitieron sostener una práctica y una conceptualización acerca de la posibilidad de tomar la palabra. Esta toma de la palabra se identificó en sentido real y metafórico, y se documentó en situaciones de intervención en la institución hospitalaria.

Fue a partir de ese encuentro con las familias que se fue conformando un equipo interdisciplinario denominado "Lecturas en diversidad" (profesionales de diferentes disciplinas en el hospital). El concepto de diversidad alude a las características de la población que asiste al servicio de pediatría, ya que algunas familias provienen de países limítrofes y de varias provincias de Argentina. Todo esto quedó representado en el uso del plural en los términos "lecturas" $y$ "escrituras", tal como figura en el nombre del proyecto. En las prácticas vinculadas a la lectura y la escritura que se desarrollan en este espacio, se trabaja con la palabra, con textos, con el cuerpo, con música, con plástica, con sonidos, silencios, ruidos y muchos otros soportes de expresión. Diversidad también alude a una concepción de trabajo divergente, que no busca disolver las tensiones y contradicciones entre enfoques, sino dar cuenta de una construcción transdisciplinar, integrada por psicólogas, musicoterapeutas, docentes de letras y de educación (atención temprana, primaria e inicial), médicos, enfermeros, trabajadores sociales y usuarios del sistema de salud. Además, los distintos miembros del equipo son de tres áreas ministeriales que tienen sus propias lógicas: salud, cultura (Programa Cultura en Barrios) y educación (Escuela Domiciliaria № 1 y Hospitalaria). Esto implica un ejercicio de armado, construcción de sentidos en común, sosteniendo al mismo tiempo las especificidades de cada disciplina y área.

\section{La Biblioteca "Lobo, ¿estás?"}

Funciona dentro de una escuela de nivel medio del barrio de Parque Patricios. Dentro de esta escuela, desde el año 2015 se alojó una sala maternal perteneciente al Programa Primera Infancia, que recibe a las hijas e hijos de las alumnas/os que estudian en la escuela media, así como otros estudiantes que vienen de escuelas cercanas. Esta sala surge con la intención de dar respuesta a la necesidad de las/los estudiantes de continuar su escolaridad secundaria, ofreciendo un espacio educativo para sus hijos/as mientras sus madres y padres están cursando.

Asimismo, las alumnas madres, alumnos padres y embarazadas reciben el acompañamiento del Programa de Retención de Alumnas Embarazadas, Madres y Alumnos Padres, para que puedan continuar con sus estudios obligatorios. La convivencia entre la sala maternal y la escuela media se entreteje en torno a la palabra escrita, leída, escuchada, en torno a la literatura. ¿Cómo pensar la literatura desde los primeros meses de vida de las niñas y niños pequeños, y cómo, al mismo tiempo, pensarla con las y los adolescentes? ¿Cómo pensar la literatura como 
ocasión de encuentro entre ambos? ¿Cómo pensar el impacto de esta relación en las y los demás estudiantes y docentes de la escuela media?

Del mismo modo, se planteó otro interrogante que surgió a partir de consultas realizadas por papás y mamás jóvenes que participaron en la experiencia: “Las/os bebés ¿leen?". En este equipo se partió de suponer que sí, en tanto consideremos la lectura en sentido amplio como construcción de significado: la niña/niño pequeño es un gran lector del mundo que lo rodea, y a partir de su propia lectura de gestos y movimientos, palabras oídas y silencios, olores y temperaturas, construye experiencia. Una lectura que comienza a significarse mediante el lenguaje y la poesía que arrulla, abraza y acaricia a los bebés desde el inicio de la vida misma. Esas son las diversas materialidades con las que se trabaja en el espacio.

\section{Prácticas de aula. Escuela pública barrial}

Esta experiencia se realiza en una escuela del barrio de Villa Lugano. La persona que lleva adelante estas prácticas $(H$.$) trabajó en la misma escuela durante 11$ años (2007-2018), lapso en el que la dirección de la escuela cambió tantas veces como una por año. Este hecho hizo que mucho de lo que en otras escuelas asume la dirección fuera distribuido entre los/las docentes. Esa cualidad del ejercicio del poder institucional (repartido, cambiante, auto-organizado) dio la posibilidad de realizar prácticas que se fueron produciendo como una construcción singular de esta escuela, donde existe la posibilidad de ensayar, realizar, pensar en conjunto, y hacerlo entre varios colegas y las familias.

También es una escuela conocida en ese barrio porque su población de estudiantes y familias enfrenta situaciones muy complejas, ligadas a dificultades para acceder a derechos básicos como vivienda, trabajo, alimentación, recreación, educación, participación política. En ese sentido, en el imaginario social del barrio es una escuela estigmatizada.

Como en otras de las experiencias presentadas, en esta escuela los estudiantes provienen en su mayoría de países limítrofes y de otras provincias. Aunque las condiciones de vida de los estudiantes son muy difíciles, en la medida de lo posible esta escuela busca garantizar el ejercicio pleno de derechos para sus estudiantes.

Con respecto a la relación entre lectura, escritura y arte, en esta escuela se realizan sistemáticamente experiencias que constituyen un proceso de construcción pedagógico-cultural colectivo, tales como "Literatura en proyectos comunitarios", "Encuentros semanales de bienvenida artística" (a través del teatro comunitario, en el que especialmente actúan juntos padres, madres, docentes y alumnos), proyectos de aula de "Re-narraciones" de leyendas e historias de la tradición popular. El análisis de H. es que el hecho de que la Dirección de la escuela haya sido asumida más por una efectuación colectiva docente que por un director/a, generó las condiciones para crear estos espacios de práctica dentro de la misma, donde familias, estudiantes, docentes y no docentes se vincularon a través de la lectura, la escritura y las artes. 
Los formatos de estas prácticas y las materialidades en que se construyen son diversos y han variado de experiencia en experiencia, de año en año y de grupo en grupo. Algunas constantes, sin embargo, son: el interés por integrar varias formas del arte visual a la lectura y escritura escolares (a través de obras de arte visuales de artistas plásticos o de que los mismos niños produzcan dibujos, pinturas, croquis y diseños); la posibilidad de interpretar dramática y corporalmente algunos textos escritos o leídos en el aula o preparados por las familias y el equipo de la escuela, como obras de teatro al inicio del día escolar; la insistencia en realizar actividades que permitan vincular el adentro de la escuela con el afuera, sea a través de convocatorias a participar, de hacer murales o actividades en calles del barrio o a través de incorporar lo cotidiano de las familias a la tarea del aula. El acento en la materialidad del arte visual y sus formatos y medios distintos (acuarelas, dibujos, tinta, collage, témperas, pintura de paredes, croquis en proporción), da a la propuesta pedagógica una posibilidad de experimentación y juego con lo concreto material, que muchas veces se pierde de vista en ámbitos de salud o escolares, tanto en las escuelas primarias y secundarias como en los contextos de educación terciaria o universitaria.

\section{Contramaratón de lectura}

Surge de la propuesta de un equipo de bibliotecarios y docentes de una escuela normal superior en el barrio de Almagro. Esta escuela cuenta con distintos niveles educativos: inicial, primario, secundario y profesorados de educación inicial y primaria. El edificio es compartido con otras dos instituciones de nivel secundario, y en el segundo subsuelo se encuentra la biblioteca, que comparte el lugar con las instituciones mencionadas.

El nombre de la experiencia se define en tensión con otras experiencias que proponen realizar "maratones de lectura", ligadas a la competencia individual. Frente a esta significación, el Contra-maratón se propone como una invitación colectiva de lecturas singulares, personales y compartidas, propiciando una apropiación sensible de la experiencia de leer y escribir.

La lectura no es una carrera ya que precisa de tiempo, de lectores/as y de intercambios. Por esto se decidió que duraría más de un día, sin que esto fuera fijado de antemano, por lo cual en las distintas ediciones (realizadas entre 2014 y 2019), la duración fue variando entre una semana y un cuatrimestre. Durante ese tiempo se realizan propuestas de lectura y escritura que invitan a los participantes a detenerse, a leer en otro ritmo.

En estas experiencias, el equipo de la biblioteca coordina la actividad junto con maestros/ as, profesores de distintos niveles y estudiantes del profesorado que se acercan con sus propuestas de manera personal o como parte de una cátedra. En este sentido, se convierte en la ocasión de que la escuela sea un espacio de encuentro a partir de la lectura y de dinamizar los roles establecidos ya que, en realidad, todos quienes participan pasan a ser lectores y no tiene importancia si son docentes, alumnos, adultos, niños o adolescentes, sino que el eje está puesto 
en leer, disfrutar de la lectura, socializar lo que se va aprendiendo y proponer formas novedosas de hacerlo.

Las prácticas de lectura y escritura del Contramaratón son fundamentalmente literarias y se producen en el cruce de distintos lenguajes y soportes. El lenguaje verbal aparece en la lectura en voz alta de texto escrito, puesto en voz de distintos modos; la escritura, en papel devenido juguete, como en los aviones poéticos o las preguntas del libro de Neruda, o impresas en carteles que luego quedarían en las paredes; el lenguaje audiovisual, que combina distintos lenguajes junto con el oral y escrito, la música, lo teatral que se produce en estas escenas, son algunos de los ejemplos de estos cruces.

\section{Interpretar el potencial transformativo en la subjetividad y las instituciones} Con la intención de poner en discusión, a través de las experiencias relevadas, la noción de potencial transformativo, propósito ya señalado en un inicio, hemos presentado en la sección anterior una descripción analítica de cada experiencia, situando cada una en su contexto y complejidad y tomando en cuenta los núcleos de sentido que explicamos en la sección metodológica. Aquí nos adentramos en un análisis interpretativo, detallando primero puntos en común, para luego presentar algunos resultados en relación con el siguiente interrogante ¿De qué modos (contextualmente situados) estas experiencias sostienen y desarrollan un potencial de transformación, tanto en el grupo que la lleva adelante como en relación con el contexto organizacional en que se aloja?

En primer lugar, en relación con los puntos en común, quienes se identifican como sujetos de las prácticas incluyen tanto a quienes las diseñan e implementan como a sus destinatarios, configurando así un conjunto heterogéneo conformado por usuarios del sistema de salud y educativo público (que van desde bebés hasta estudiantes del nivel terciario), familias (con configuraciones diversas), docentes, psicopedagogas, bibliotecarias, entre otros. En principio, esta relación heterogénea entre los usualmente referidos como "usuarios y profesionales" se da en el interior de cada experiencia. Sin embargo, en nuestro trabajo constatamos que muchos de estos espacios se conocen entre sí, se referencian y se comunican. A través de la puesta en marcha de este proyecto que presentamos aquí, además, se construyeron otros modos de intercambio sistemático entre experiencias que, como dijimos al inicio, también tienen la particularidad de que quienes las llevamos adelante asumimos simultáneamente roles diversos, por ejemplo, participamos en experiencias sociocomunitarias en nuestros barrios en relación con la lectura y la escritura y también somos docentes en los distintos niveles escolares (primario, secundario, terciario y universitario); o bien, trabajamos en dependencias públicas (salud, educación hospitalaria, trabajo social barrial) y llevamos adelante experiencias de lectura y escritura en esos ámbitos y simultáneamente en nuestras comunidades. El hecho de que la definición de los sujetos involucrados abarque esta variedad permite producir una noción de sujeto colectivo que 
se genera a partir de un encuentro entre personas, más allá de los roles formales que se ocupan en estas prácticas (docente, estudiante, médico, paciente). En el interior de las organizaciones (hospital o escuela), este hecho genera una interpelación de los modos discursivos habituales, por ejemplo, modos estereotipados de establecer conversaciones médico-paciente, maestroalumno, familia-institución pública de salud o escolar.

En segundo lugar, siguiendo con las cuestiones similares entre experiencias, en cuanto a las materialidades, lenguajes y formatos involucrados en las prácticas, observamos una gran diversidad: oralidad, silencios, gestualidad, lenguaje visual, poesía, música, plástica. En tanto la lectura y la escritura se producen de forma multitextual, estas experiencias van reconfigurando la misma noción de leer y escribir en su quehacer.

Finalmente, en cuanto a la infraestructura, identificamos que las experiencias muchas veces se configuran a partir de la confluencia de diversos programas estatales y están inscritas en una compleja red de vínculos y pertenencias institucionales, entre las cuales parecen brindar prácticas que atraviesan esos diversos espacios a través de un ejercicio particular con la lectura y la escritura. Por lo tanto, estamos en presencia de modos novedosos de llevar adelante las políticas públicas ya que los sujetos colectivos que las efectúan las van redimensionando en su quehacer diario.

\section{Transformación de la subjetividad a través de la lectura y la escritura}

En las experiencias analizadas, las prácticas de lectura y escritura, están ligadas a la posibilidad de construir narrativas, imaginar otros mundos posibles, inaugurar otras formas de sensibilidad en las cuales el cuerpo ocupa un lugar central. Se identifican por una apertura a lo incierto y una escucha y lectura de lo que ocurre en cada momento particular. En la experiencia "Lecturas en diversidad", esta forma de trabajo se constituye como un modo de abordaje donde se abre a la escucha y se invita a leer y a jugar, pensando como punto de partida que todos somos otros y buscando deconstruir la noción de un otro estigmatizado. El juego plantea un pacto implícito vinculado a desplegar diferentes miradas, acciones, movimientos, creaciones, ante una realidad que podría parecer establecida: profesionales / familias, salud / enfermedad; argentinos / extranjeros, entre varios de los esquemas referenciales interpretativos y de sentido común que tienen lugar en el hospital. A través de las actividades se abren posibilidades de múltiples lecturas en un mismo acontecimiento, porque se trabaja con niños/as, familias y profesionales en conjunto, narrando o re-narrando lo que en un principio no está puesto en palabras, o aquello que puede surgir como inesperado. Esto exige una escucha activa por parte de quienes coordinan el espacio, una apertura a lo sensible para captar lo que está ocurriendo en ese momento y poder leerlo en las interacciones para no interferir con su despliegue. El equipo denomina a esta práctica "la posibilidad de trabajar con lo que acontece".

Algo similar se plantea en la escuela de $\mathrm{H}$, al referirse al trabajo que realiza con sus estudiantes y las posibilidades de abrir un plano ligado al juego y la imaginación vinculado a la narración, 
ya que se identifica que la lectura produce una "calma activa que latía despabilada en los niños/ as, que estaban idos en la imaginación, una función interesante para la literatura en la escuela". En palabras del maestro que llevó adelante esta experiencia, "parecía que viajaban navegando, yéndose lejos, y se reían, comentaban, se emocionaban, compartían. Afloraba el jardín de la sensibilidad con todos sus pétalos. Funcionaba también porque ninguno preguntaba: "Profe ¿para qué nos leés este cuento?" o “De qué me sirve escuchar literatura?" Ese funcionamiento no hace falta medirlo. No se cuantifica la emoción. Es imposible ponerle número a la sensibilidad o a la transformación de la conciencia. Aunque no tenemos registro empírico, sabemos que algo pasó, una cosa sucedió, un evento aconteció. La potencia devino acto.

De allí que la lectura es una interpretación posible de lo que está ocurriendo, tanto por parte de quienes coordinan la actividad como de los niños y niñas que están participando. De esta manera, aparece la apertura de otro plano de la realidad donde se habla y se juega, donde se puede ir y venir.

Los y las integrantes de "Lecturas en diversidad" se refieren a estos estados como "ensoñaciones compartidas". La apuesta de estas experiencias es animarse a trabajar en ese plano que se asemeja a la definición que el escritor Julio Cortázar hace de lo fantástico:

hay como pequeños paréntesis de la realidad y es por ahí, donde una sensibilidad preparada a ese tipo de experiencias siente la presencia de algo diferente, siente, en otras palabras, lo que podemos llamar lo fantástico. [...] las pautas de la lógica, de la causalidad del tiempo, del espacio, todo lo que nuestra inteligencia acepta desde Aristóteles como inamovible, seguro y tranquilizado, se ve bruscamente sacudido, como conmovido por una especie de viento interior que los desplaza y los hace cambiar (Cortázar, 1982).

Acontece allí un encuentro que no persigue efectividades sino la expresión de subjetividades. Un acontecimiento que podemos percibir como belleza, ya que cuando una experiencia es sensible conmueve cuerpo y emoción, contacta con lo vital, nos sorprende, y deviene acto de belleza (Martínez, 2016).

En consonancia con el plano lúdico aludido, el Contramaratón aparece como constante en las distintas ediciones de esta experiencia. El aspecto lúdico es central en las prácticas de lectura y escritura propuestas y muchas veces se combina con la circulación por espacios no previstos en la institución escolar. Estos aspectos adquirieron un relieve singular en el cierre de la edición 2016, con la experiencia "Aviones poéticos", que convocó también al trabajo colectivo. Entre estudiantes del profesorado y bibliotecarias armaron los avioncitos que en un canasto fueron llevados al aula de séptimo grado, junto a una selección de libros de poesías. Se les propuso a los chicos/as que leyeran y escogieran estrofas, coplas, limeriks, colmos o algunos versos que les gustaran, para escribirlos en los aviones de papel y compartir luego con los demás chicos de la escuela. Con los aviones poetizados, los y las chicas de séptimo accedieron a la terraza del segundo piso, donde transcurre el recreo de la secundaria, y desde ahí se esperó el momento 
en que primero y segundo grado salieran al recreo para lanzar los aviones. Algo sorprendidos, pero entusiasmados y a los gritos, los y las pequeñas recibieron en el patio los aviones $y$, aunque al principio no percibieron los textos que portaban, luego de un rato comenzaron a leer en grupos la poesía que había llegado en vuelo desde la terraza. Estas experiencias lúdicas, donde el cuerpo está puesto en juego, amplían el espacio simbólico y también el real, ya que la escuela puede habitarse de otras maneras.

En la Biblioteca "Lobo ¿estás?", al tratarse de niñas y niños muy pequeños y sus padres y madres, la literatura está vinculada al surgimiento de las primeras palabras y a la construcción de identidades de alumnas madres y alumnos padres en relación con un doble rol: ser madres y padres adolescentes y al mismo tiempo estudiantes. A este ejercicio de sus roles se suma la experimentación de una relación diferente con los libros y las imágenes, rememorando vivencias de sus propias infancias y construyendo nuevas experiencias en las que la lectura los interpela y conmueva de otras formas posibles. La palabra envuelve narrativamente el silencio que introduce el diálogo, la cercanía del vínculo que acompaña y sostiene amorosamente, la metáfora que da lugar al encuentro y al reconocimiento de uno mismo a partir del otro.

Esta apertura de otro plano de la realidad vinculado a la ensoñación, al estar idos, a un encuentro amoroso, a lo lúdico, es lo que habilita la posibilidad transformadora de la subjetividad en estas experiencias. H. habla de un proceso emancipatorio vinculado a la lectura de literatura, ya que hay figuras de la actividad intelectual que la lectura propicia, por ejemplo, la anticipación (una predicción que se realiza articulando los indicios recolectados del texto) o la atribución de sentido (una interpretación, una producción, una creación, que es producto de la actividad de lectura colectiva).

En definitiva, leer es asumir un desafío. Hay algo a resolver, un desciframiento, un enigma -aunque no se haya planteado como tal-. Leer es ubicarse en una posición activa de trabajo y producción artesanal, no mercantil. La interpretación es trabajo, es confección, es obra. Y su producción es conjunta: se lee con los demás y gracias a los demás. Es la trama del taller comunitario. Colocar en esta posición es emancipador, porque el sujeto así sabe que sabe, es consciente de su capacidad de entender y producir. No recibe pasivamente, como si fuera objeto, como si fuera depositario de una conciencia exterior. Se inserta así en el escenario de la historia como protagonista, como actor principal con un libreto a escribir. La enajenación es la separación del deseo. Un enajenado es quien no tiene contacto con sus ganas, con las fuentes que le proporcionan placer. La literatura también repara ese vínculo, por eso es des-enajenante.

En "Lecturas en diversidad" también hay una relación con lo emancipatorio. Lo que se intenta es crear un espacio de libertad para que cada uno se narre desde otro lugar, tal vez diferente al lugar en el cual los demás lo ubican. A este proceso se lo denomina en el grupo "el advenimiento de lo subjetivo", es decir, un devenir de cada una de esas singularidades, de esas historias y de sus genealogías también. De esta manera, la narración se vincula con la subjetividad y con la posibilidad de construcción de otros relatos, tal vez inesperados. 
En “Lobo ¿estás?" se explicita el carácter transformador de lo literario: se abre un lugar para que la narrativa y la poética circule, habite esos cuerpos, abra horizontes ficcionales para que la vida misma se transforme, o al menos lo intente. En la construcción del camino lector, la Biblioteca tiene el deber de ofrecer distintos espacios y alternativas para que las y los estudiantes puedan vincularse con la palabra literaria en todos sus sentidos y puedan trascender las dicotomías entre leer por obligación académica o leer por placer. De esta manera, la Biblioteca “Lobo, ¿estás?" pretende trascender esta dicotomía, tensionar la idea de la existencia de una literatura para adultas y adultos y otra literatura para bebés, niñas y niños, y reivindicar el lugar de la imagen en las lecturas de los adolescentes y adultos.

El diseño del Contramaratón como una práctica situada, dispuesta para actuar con otros y que otros actúen en esa situación, abierta al azar y a la extrañeza de los lectores, interpela a docentes y futuros maestros como mediadores, a la vez que les demanda pensar en los chicos no solo como meros receptores, sino como constructores de sentidos propios y colectivos, hacedores mismos de los encuentros.

Consideramos que la experiencia lúdica y los recorridos espaciales relatados significan una ampliación de la experiencia y cambios de posición de los sujetos. Para dar cuenta de la multiplicidad de formas de aprender a leer por parte de quienes asisten a estas experiencias, narramos a continuación una situación que tuvo lugar en una de ellas:

Una señora de unos 60 años vino con su nieto al servicio. Él entró a la biblioteca y dijo "jacá toqué por primera vez un instrumento! ahora tengo un ukelele". Fanático de la historia argentina, se llevó en préstamo el comic de Pigna y su abuela me empezó a contar que le encanta leer. La invité a que explorara la biblioteca y se entusiasmó con un libro que se llevó también en préstamo. Ahí me dijo que no había ido a la escuela, que solo iba para comer, que había sido muy pobre de chica y como tenía "otros pensamientos" se escapaba de las clases. Pero que aprendió a leer sola, viendo revistas. Se dio cuenta de que una letra al lado de la otra formaba palabras y a deducir lo que decían por el contexto. Le pregunté si le interesaba algún libro de poesía y me dijo "no, gracias, ya leí mucho de eso, prefiero este". Y se llevó una novela.

Por lo dicho hasta aquí, sostenemos que estas experiencias permiten visibilizar los modos (contextualmente situados) en que se sostienen y desarrollan potenciales de transformación. Asimismo, permite sostener la noción de que dicha transformación es una puesta en acto permanente, y con esa noción podemos indicar así también el potencial performativo de este tipo de posicionamiento educativo, artístico y organizacional.

\section{Interpelar el contexto institucional}

Tal como mencionamos más arriba, la mayoría de las experiencias se alojan en tramas institucionales complejas por tratarse de diferentes pertenencias (en relación a que sus puestos de trabajo se sustentan a través de diferentes programas o dependencias del Estado) e inclusive 
distintas formaciones y prácticas disciplinares. Lo que tienen en común es que en alguna medida logran transformar tanto al propio equipo que las lleva adelante como a algunas lógicas hegemónicas presentes en las instituciones que las alojan. Por ejemplo, "Lecturas en diversidad" se contextualiza dentro del sistema de salud hegemónico. Consideramos que la misma tiene efectos de transformación de la cultura hospitalaria: se cambia la narrativa intraconsultorio a través de que, quienes concurren al servicio y pasan por las actividades que se realizan en la biblioteca, comienzan a posicionarse de otro modo frente a los médicos y valoran más su propia narrativa. Esto es un trabajo que se ha logrado luego de más de 20 años, que es el tiempo que esta experiencia lleva alojada en este servicio de salud. En la actualidad ya hay un reconocimiento de que el espacio tiene que ver con el modo específico de atención en este servicio de salud. A pesar de esto, en algunas ocasiones se identifica también que las familias que concurren al hospital, y en particular al servicio ambulatorio de pediatría, perciben dos espacios diferenciados: el del consultorio, con sus propias formas de interacción, y el de la biblioteca. La pauta de esto nos la dio un niño que estaba haciendo una actividad en la biblioteca y que al escuchar que lo llamaba el médico desde el consultorio porque había llegado su turno, nos dijo "me voy al otro hospital".

En el caso de las prácticas de aula de la escuela de H., al mismo tiempo que se interpela el aislamiento en la escuela primaria se transforma la subjetividad docente. Uno de los temas que siempre preocupó a $\mathrm{H}$. es el que vincula el aprender escolar con el aprender en la vida cotidiana, tanto para los educadores como para los alumnos y sus familias. En este sentido, H. buscó conformar grupos de trabajo y reflexión sobre la práctica docente o participar de momentos de encuentro esporádicos con otros trabajadores del arte y la cultura, preguntándose: ¿qué relación podemos encontrar entre aprender a leer textos y aprender a leer el mundo? ¿Qué aspectos de la lectura se vinculan con el arte, el disfrute estético y la construcción de modos de interpretar (obras de arte visual, literario u otras cuestiones) que permitan expandir lo conocido? A partir de esas preguntas, se contactó con otros colegas de la misma escuela ya que las instituciones que habitamos, por su estructura organizativa, tienden a aislarnos. Específicamente en relación con la lectura, escritura, arte y escuela, $\mathrm{H}$. y sus colegas "de la primaria" realizaron varias experiencias que él describe como "un proceso de construcción pedagógico-cultural colectivo".

En cuanto a la Biblioteca "Lobo ¿estás?" la presencia de la sala maternal en la escuela media convoca al encuentro. Es una apuesta a la convivencia institucional centrada fundamentalmente en torno a la lectura. La biblioteca concreta uno de los propósitos del proyecto educativo de la sala maternal, que es trabajar de manera articulada y en conjunto con la escuela media. Este propósito se asume con intención y convicción de generar espacios de trabajo que involucren y reúnan a docentes de diferentes niveles educativos, en este caso, inicial y media, sujetos pedagógicos de muy diferentes edades y, además, un entramado de relaciones que vinculan maternidades, paternidades, escolaridad y crianza. 
En cuanto al Contramaratón, la posibilidad de tomarse el tiempo para leer de diferentes modos cuestiona una idea de lectura que el maratón representa de algún modo: leer cantidades de libros para ganar una competencia. En otro sentido, la construcción colectiva del proyecto, los movimientos en relación con roles fijos, la posición activa de distintos participantes, la circulación por espacios no habituales, producen una interpelación a lo que puede hacerse costumbre en una institución, a los lugares rígidos, a la pérdida de sentidos. La organización del Contramaratón intenta ser siempre una invitación a la lectura y no una imposición, por eso dedica especial atención a las actividades de apertura, buscando que tengan algo de "apartamiento" del orden, como dice Graciela Montes (2007), con el objeto de generar el deseo, la provocación o el desconcierto.

\section{Resultados y nuevos interrogantes}

En la sección anterior hemos dejado planteadas algunas cuestiones relevantes para continuar profundizando en esta perspectiva de análisis que toma las prácticas de lectura y escritura como prácticas situadas que, bajo ciertas condiciones, habilitan a los sujetos que participan en ellas a construir sentidos múltiples, nuevas lecturas de la realidad, nuevas narrativas, posicionamientos diferentes frente a lo dado. Estos movimientos intersubjetivos, que a su vez interpelan ciertos modos de lo social en las instituciones y organizaciones que las alojan o con quienes interactúan, son considerados procesos continuos, indeterminados y abiertos. Al mismo tiempo, consideramos que la propia reflexión sobre la práctica, la descripción y análisis de las mismas, permite una aproximación teórica a las relaciones entre lectura, escritura, creación organizacional y cambio. Profundizaremos aquí algunos aspectos a poner en discusión.

Como ya hemos dicho, las prácticas de lectura a las que nos referimos asumen deliberadamente que leer implica una actividad interpretativa. En el análisis de las experiencias aparecen diversos sentidos del término lectura o leer que podemos relacionar con el marco teórico de los NEL, que en su postulación primera siguen a Freire: leer el mundo. Se asume y se busca potenciar una fuerte actividad de los lectores en la construcción de sentidos, no solo de textos escritos y de imágenes, sino también de lecturas de la realidad, de los acontecimientos. Leen lo que acontece quienes acompañan a los lectores más jóvenes, niños o bebés. Se pone en juego algo del orden del detenerse, mirar, estar abiertos, sostener, escuchar al otro, lo cual produce lecturas sensibles. En este sentido, las escenas recuperadas en las entrevistas provienen de una lectura abierta a dejarse sorprender.

Las lecturas se producen colectivamente. Se lee con otros distintos, en la lógica de una construcción colectiva donde se incorporan y articulan una pluralidad de sentidos diversos. "Se lee con los demás, gracias a los demás" dice el maestro $\mathrm{H}$.

Los cruces y encuentros de participantes diversos que enriquecen las lecturas aparecen en las distintas experiencias. En "Lecturas en diversidad" se sostiene desde su formulación: hay 
construcciones de sentidos en común sosteniendo las especificidades. Así, profesionales que provienen de diversas disciplinas y de ministerios con lógicas distintas, niños y niñas con sus cuidadores que provienen de distintas culturas, crean algo novedoso dentro del hospital y en las actividades barriales que luego se desarrollaron a partir de la experiencia. El Contramaratón se define como construcción colectiva en la que se encuentran sujetos de distintos niveles educativos y con distintos roles. En "Lobo, ¿estás?" confluyen distintas instituciones y programas, adultos con roles diferentes y formación docente, bebés y adolescentes madres o padres con tensiones propias de los marcos institucionales con los que interactúan.

En algunas de estas experiencias ha aparecido más de una vez la alusión a La gran ocasión. La escuela como sociedad de lectura, un ensayo dirigido a maestros, bibliotecarios y otros mediadores de lectura que sostiene muchas de estas convicciones. Allí, Graciela Montes (2007), en lenguaje poético, parte de una definición de lectura como "codicia de sentido" y la posición del lector como de "insubordinación y deseo". El subtítulo anticipa que la escuela es una ocasión para que se produzcan lecturas en este sentido. Ya no aparece el lector solitario, romantizado, sino la oportunidad del intercambio, de la construcción en la diferencia de voces. Estas concepciones de lectura ponen en tensión una tradición escolar de comprensión estrecha que busca que los lectores "digan de memoria" o"repitan con sus palabras" el texto. También desafían otras versiones más contemporáneas de la misma posición, que se manifiestan en las evaluaciones internacionales. Y por último, también desafían y tensionan una cierta versión divulgada de la "libertad del lector" que se confunde con un "subjetivismo blando" (Larrosa, 2008) y que llevaría a una postura individualista extrema del tipo "la lectura es subjetiva".

Por fin, otro aspecto que aparece claramente es la importancia de lo lúdico como medio para que se produzcan nuevas lecturas por su potencia como lenguaje simbólico y por su relación con el arte. El juego participa del carácter ficcional de la literatura, abre la posibilidad de entrar a otro mundo y a otro plano distinto que necesita un tiempo y una sensibilidad diferente. La literatura es valorada como lectura privilegiada por abrir a mundos ficcionales. Las cuatro experiencias explicitan el valor del texto literario. Los encuentros colectivos donde el juego, la literatura y otras artes producen lecturas y escrituras, sostienen la emergencia de la palabra propia y de los y las demás, reconociendo autorías diversas y múltiples, nuevas narrativas, contar de otro modo y posicionarse de otro modo.

Hasta aquí, hemos mencionado algunas cuestiones relevantes que surgen del análisis de las cuatro experiencias presentadas. Podemos ratificar que nuestra perspectiva, que toma las prácticas de lectura y escritura como construcciones de transformación en procesos continuos, indeterminados y abiertos, permite también identificar las transformaciones subjetivas e intersubjetivas, a la vez que nos alertan acerca de las transformaciones en las organizaciones que les dan cabida. Por tanto, dejamos anotado que este marco permite una aproximación teórica a las relaciones entre lectura, escritura, creación organizacional y cambio sociopolítico. 
En nuestro trabajo existe evidencia que da cuenta de que las transformaciones subjetivas que producen este tipo de actividades están interpelando estructuras sociopolíticas. Esta evidencia podría pasar desapercibida si no apeláramos a un marco de análisis como el construido para la interpretación de estas prácticas. Por ejemplo, situaciones donde queda expuesto el movimiento que se va produciendo en interacción, de modo tal que permite a los sujetos involucrados construir modos de vinculación diferentes a los que habitualmente son sometidos, que en los casos que estudiamos suelen ser modos de violencia institucional y estructural naturalizados. Sostenemos que el enfoque que adoptamos para el análisis permite tomar en cuenta esta evidencia como tal, y no como una anécdota o circunstancia casual.

De todas maneras, entendemos que una limitación de nuestro estudio es que, para realizar un trabajo etnográfico de largo término que permitiera continuar corroborando cómo ocurren dichas interpelaciones a las violencias estructurales, necesitaríamos desarrollar un estudio en otros tiempos y contando con recursos para sostener un relevamiento en profundidad. Asimismo, las condiciones actuales de pandemia han puesto en severa restricción ese tipo de metodologías. Si bien estamos generando otros métodos para continuar el trabajo realizado hasta aquí, el mundo como lo habíamos conocido está cambiando y estamos aún en procesos de estabilización de formas diferentes de producción de acción transformadora y conocimientos al respecto.

Un relevamiento en profundidad y en tiempos más largos nos permitirá, dentro de los nuevos paradigmas que generemos para producir conocimiento colectivo, incorporar otro material de observación directa por parte de los diferentes miembros del equipo, lo cual estimamos importante por dos motivos: el primero, porque nuestra hipótesis es que podríamos recoger una mayor cantidad de evidencia acerca de cómo ocurren las transformaciones e interpelaciones al sistema dominante, inclusive -o más aún- en momentos donde la pandemia ha dejado al descubierto situaciones de injusticia profundas que en otros tiempos podían estar menos visibles; y el segundo es que podríamos inclusive comprender cómo dichas transformaciones tal vez ocurran en otros ámbitos diferentes y desafiantes, pero con situaciones similares a las observadas a través de estas experiencias.

Asimismo, y vinculado con lo anterior, otra limitación que señalamos es que se precisa de tiempos largos para establecer vínculos como los que hemos logrado en este trabajo de indagación conjunta, tiempos que a veces no están disponibles para todos/as y que suelen obtenerse por un alto grado de exposición continuada a muchas horas de trabajo. Si bien esto es común a los trabajadores de espacios públicos como somos todos los autores de este artículo, no por común deja de ser difícil y -en ciertos momentos- alienante. De esta manera, la voluntad de pensar en conjunto y teorizar a partir de nuestras experiencias ha superado las dificultades de tiempo y espacio, aun en contextos de alta vulnerabilidad. Nos parece precario que un trabajo de conceptualización como éste dependa de nuestras voluntades. 


\section{Referencias}

Barta, J.; M. E. Brenner (2009). Seeing with Many Eyes: Connections between Anthropology and Education. En Greer, B.; S. Mukhopadhyay; S. Nelson-Barber; A. Powell (eds.). Culturally Responsive Mathematics Education. Nueva York: Routledge, Taylor \& Francis, 85-110.

Barton, D.; M. Hamilton (1998). Local Literacies: Reading and Writing in One Community. Londres: Routledge.

Bonin, J. E. (2018). Discourse and Mental Health: Voice in Equality and Resistances in Medical Settings. Londres: Routledge.

Brilliant-Mills, H. (1993). Becoming a Mathematician. Linguistics and Education, 5, 301-334.

Broyles, Y. (1994). El teatro campesino. Theater in the Chicano Movements. Austin: University of Texas Press.

Butler, J. (2017). Cuerpos aliados y lucha política. Hacia una teoría performativa de la asamblea. Buenos Aires: Paidós.

Cambre, M. C. (2013). Inmanence \& Collage Heuristics. Visual Arts Research, 39(1), 70-89.

Céspedes, M.; C. Guarini (1995). Mirar las mismas cosas de una manera diferente. En Toledo, T. (comp.). Cine-Ojo. El documental como creación. Buenos Aires: Universidad del Cine / Filmoteca Generalitat Valenciana, 11-13.

Córtazar, J. (1982). El sentimiento de lo fantástico. Conferencia dictada en la UCAB.

Craviotto, E.; A. I. Heras; J. Espíndola (1999). Cultures of the Fourth Grade Bilingual Classroom. Primary Voices, 7(3), 25-36. NCTE.

Fernández, A. (2016). Lectores de signos, lectores de guiños: el ensayo curatorial como forma. Estudios Curatoriales Pensar con Imágenes, (Número Especial), 91-111.

Floriani, A. (1993). Negotiating what Counts: Roles and Relationships, Text and Context, Content and Meaning. Linguistics and Education, 5, 241-273.

Frankenstein, M. (1989). Relearning Mathematics. A Different Third R-Radical Maths. Londres: Free Association Books.

Fránquiz, M.; J. Green; E. Craviotto (1993). What is Meant by Quality of Teaching? Towards a Social Constructionist Perspective in Teacher Education. Education Research and Perspective, 20, 13-24.

Freire, P. (1983). The Importance of the Act of Reading. Journal of Education, 165(8), 5-11.

Frisch, P.; N. Stoppani (comps.) (2014). Hacia una pedagogía emancipatoria en nuestra América. Argentina: Ediciones del CCC.

Gee, P. (1986). Oralidad y literacidad: del pensamiento salvaje a ways with words. En Zavala, V.; M. Niño-Murcia; P. Ames (eds.). Escritura y sociedad: nuevas perspectivas teóricas y etnográficas. Lima: Red Desarrollo de las Ciencias Sociales del Perú, 23-55.

Heras, A. I. (1993). The Construction of Understanding in a Sixth-Grade Bilingual Classroom. Linguistics \& Education, 5(3-4), 275-279. 
Heras, A. I. (1995). Living Bilingual, Interacting in Two Languages: An Ethnographic and Sociolinguistic Study of a Fourth-Grade Bilingual Classroom. Tesis doctoral. Santa Bárbara: Universidad de California.

Heras, A. I. (1999). Taking Action with Family and Community Members: Critical Pedagogy as a Framework for Educational Change. Advances in Confluent Education. Stamford, Connecticut: JAI Press Inc., 73-107.

Heras, A. I.; E. Craviotto (2001). Mediating Different Worlds: Bicultural Students at School. En Goodman, W. (comp.). Living and Teaching in an Unjust World. New Perspectives on Multicultural Education. Portsmouth: Heinemann, 91-99.

Heras, A. I.; J. Green (2010). Identidades y políticas públicas educativas. Las consecuencias de cambiar de una comunidad bilingüe a inglés como única lengua de instrucción. En López, G.; C. Pérez. Discursos e identidades en contextos de cambio educativo. México: Plaza y Valdés, 154-195.

Heras, A. I.; Miano, A. (2014). Sociolingüística y etnografía. Análisis de interacciones en la Mesa de grupos autogestionados. La Trama de la Comunicación, (18), 251-271.

Heras, A. I.; D. Burin; T. Di Leo; C. Durañona; M. Jaureguiberry; A. Miano; M. Pacheco; M. RocCo, en colaboración con J. Flury; M. Lamacchia; P. Medrano (2014). La autonomía como proyecto: Procesos de reflexión deliberada en experiencias de autogestión. Pueblos y Fronteras, 8(16), 56-91.

Heras, A. I.; Miano, M. A. (2018). Street Children's Narrative Accounts: Availability, Access and Transposing across Semiotic Systems. XIX Congreso Internacional de Sociología. Toronto.

Hirsch-Dubin, F. (2009). Mayan Elders, Mayan Mathematics, and the Weaving of Resistance in Maguey Bag Production. The Journal of Mathematics and Culture, 4(1), 63-83.

Kalman, J. (2003). El acceso a la cultura escrita: la participación social y la apropiación de conocimientos en eventos cotidianos de lectura y escritura. Revista Mexicana de Investigación Educativa, 8(17), 37-66.

Larrosa, J. (2008). Leer (y enseñar a leer) entre las lenguas. Veinte fragmentos (y muchas preguntas) sobre lenguaje y pluralidad. Barcelona: Trotta.

López-Bonilla, G.; G. Tinajero; C. Pérez (2006). Jóvenes, currículo y competencia literaria. Revista Electrónica de Investigación Educativa, 8(2).

López-Bonilla, G. (2013). Prácticas disciplinares, prácticas escolares: Qué son las disciplinas académicas y cómo se relacionan con la educación formal en las ciencias y en las humanidades. Revista Mexicana de Investigación Educativa, 18(57), 383-412.

Martínez, J. (2016). Intervenciones musicales e instauraciones sonoras en sala de espera del Servicio de Pediatría de HGAT, como parte del equipo Promoción de las lecturas. En: Jornada Girapalabras. Buenos Aires: ALIJA. 
Marucco, M. (comp.) (en prensa). Haciendo caminos. Diez años de reflexión sobre la práctica docente. Argentina: Ediciones del CCC.

Miano, M.; A. I. Heras (2015). Imágenes y narración: análisis de un espacio pedagógico con niñas y niños en situación de calle. Ciencia, Docencia y Tecnología, 26(50), 161-187.

Miano, M.; A. I. Heras (2018). Niñas y niños toman la palabra: el potencial formativo de la narración. RLCSNJ, 16(2), 979-994.

Montes, G. (2007). La gran ocasión: La escuela como sociedad de lectura. Buenos Aires: Plan Nacional de Lectura, Ministerio de Educación Ciencia y Tecnología.

Rockwell, E. (1992). Los usos magisteriales de le lengua escrita. Nueva Antropología, 12, 43-55.

Rockwell, E. (2006). Apropiaciones indígenas de la escritura en tres dominios: religión, gobierno y escuela. Cultura Escrita y Sociedad, 3, 161-218.

Rockwell, E. (2012). Cultura escrita, historias locales y otra lógica ciudadana: relatos de los vecinos mayores de Cuauhtenco, Tlaxcala. En Calderón, M. A.; E. M. Buenabad (eds.). Educación indígena, ciudadanía y Estado en México: Siglo XX. México: Benemérita Universidad Autónoma de Puebla, 93-118.

Santa Barbara Classroom Discourse Group (1992). Constructing Literacy in Classrooms: Literate Action as Social Accomplishment. En Marshall, H. (ed.). Redefining Student Learning. Norwood: Ablex, 119-150.

Santa Barbara Classroom Discourse Group (1995). Two Languages, One Community: An Examination of Educational Opportunities. En Macías, R.; R. García Ramos. Changing Schools for Changing Students: An Anthology of Research on Language Minorities, Schools \& Society. California: Linguistic Minority Research Institute, 63-106.

Scollon, R.; S. B. K. Scollon (1981). Narrative, Literacy and Face in Interethnic Communication. Norwood: Ablex.

Scribner, S.; M. Cole (1981). The Psychology of Literacy. Cambridge: Harvard University Press.

Weissglass, J. (2001). Inequity in Mathematics Education: Questions for Educators. Mathematics Educator, 12(2), 34-39.

Weschler, D. (2016). Diálogo con Graciela Sacco. Revista Estudios Curatoriales Pensar con Imágenes, (Número Especial), 129-139.

Zavala, V. (2002). Desencuentros con la escritura. Lima: Red para el Desarrollo de las Ciencias Sociales del Perú.

Zavala, V; M. Niño-Murcia; P. Ames (2004). Escritura y sociedad: Nuevas perspectivas teóricas y etnográficas. Lima: Red para el Desarrollo de las Ciencias Sociales del Perú. 